a genuine movement of increased awareness and increased concern for animals that is occurring in many countries," he said. "There are even signs of great ape movements in countries that have not traditionally shown concern for animals."

Singer, who wrote the 1975 book Animal Liberation, a rallying cry for rights and protection for animals, said that the animal rights movement has shifted away from the image of protestors splattering blood on fur coats or disrupting animal laboratories, and into the political realm. $\mathrm{He}$ is, therefore, pleased with California's Proposition 2, which establishes that farm animals are entitled to sufficient room for roaming. "I think that [passing this referendum] shows is that the American public as a whole is supportive of at least getting rid of the worst forms of factory farming," Singer said. "I think that's where by far the largest amount of human inflicted animal suffering is." He also added that he believes the ball is rolling on animal rights much faster than expected, pointing out that more Californians voted for Proposition 2 than voted for Barack Obama, who is very popular in the state, to become president.

\section{"I suspect that primate research will slowly disappear because people have a special thing about primates."}

Indeed, the US Congress might soon establish legislation to reduce the amount of research conducted on primates. Representative Roscoe Bartlett, a Republican from Maryland, has introduced a bill that would require researchers to substitute invasive research on great apes with existing alternatives. Bartlett himself was unavailable for comment because Congress was out of session, but Lisa Wright, a spokeswoman for him, explained that the congressman is disgusted with the treatment of some of humanity's closest relatives. "Science has progressed sufficiently with tissue typing and other techniques that it is no longer necessary to utilize [...] primates, who are extremely intelligent and social animals," she said.

Yet, the passage of the bill is not a done deal. According to Justin Goodman, the research associate supervisor for People for the Ethical Treatment of Animals (PETA;
Sidebar A | Further coverage of the ethics of animal research

In EMBO reports (June 2007), see the Talking Point on the topic:

Animal rights, human wrongs?

Frank Gannon

EMBO reports 8: 519-520

Animal research: a moral science

Bernard E. Rollin

EMBO reports 8: $521-525$

The ethics of animal research

Simon Festing \& Robin Wilkinson

EMBO reports 8: $526-530$

Norfolk, VA, USA), getting laws passed for animals, even for apes, is very difficult in the USA because of a lack of public understanding of the plight of these animals. "We have no legislation for great apes right now, so it's hard to imagine giving protection to other non-human primates who people perceive to be even more different to humans anytime soon," he explained. "Because the legislation process is so slow going, we spend most of our time related to primate experimentation issues educating the public about the utter tragedy that these social and intelligent animals are being locked in cages and driven mad by confinement."

$\mathrm{N}$ otwithstanding the process, Bernard Rollin, a bioethicist at Colorado State University (Fort Collins, CO, USA) and principal architect of 1985 US legislation dealing with the welfare of experimental animals, said it has taken people long enough to stand up for animals. Yet, he also anticipates that primates will eventually be exempt from being research subjects. "I suspect that primate research will slowly disappear because people have a special thing about primates," he said. "I've heard researchers say they hate doing stuff with monkeys because they're people in monkey suits."

While the debate continues around the world, Martin and his macaques must continue to bide their time. "What I've discovered with the legal process is that it is achingly slow [...] [it has been more than] three years since my experiments were stopped, and I still don't have an answer," he said. "This has had a major negative impact on my own contribution to this important field of research, but if I were a young researcher, it would also have had a devastating impact on my training and my career prospects." For now, the balance between the rights of animals and the needs of medicine and science is neither obvious nor easy.

\section{See Sidebar A for further reading.}

\section{REFERENCES}

Estrella M (2008) Monkeys in Bremen. Lab Times [online]. http://www.lab-times.org/ editorial/e_082.html

Singer P (1975) Animal Liberation. New York, NY, USA: Avon

Staub N (2007) Their hands are tied. ETH Life international [online]. http://archiv.ethlife.ethz.ch/ articles/tages/inianimresear.html

Weatherall D (2006) The Use of Non-human Primates in Research. London, UK: Academy of Medical Sciences, Medical Research Council, The Royal Society and Wellcome Trust

Melissa Suran \& Howard Wolinsky

doi:10.1038/embor.2009.214

\title{
The secret garden
}

\section{The human digestive system is teeming with microbiotic life, but just how important are these interlopers for health and disease?}

A vast number and variety of bacteria enjoy the warm and nutrientrich environment of the human gut. Some of them are pathogens, whereas most others could be mistaken for simple hitchhikers along for the ride. Yet, it has become clear that the hitchhikers are actually paying their way; indeed, the extent of our dependence on these extragenomic microorganisms, or microbiota, for health is remarkable. The insight that our gut flora have an enormously important role in nutrition and disease has given rise to the term 'microbiome' to describe loosely all those microbial genes-and their products - that interact with our own genome 


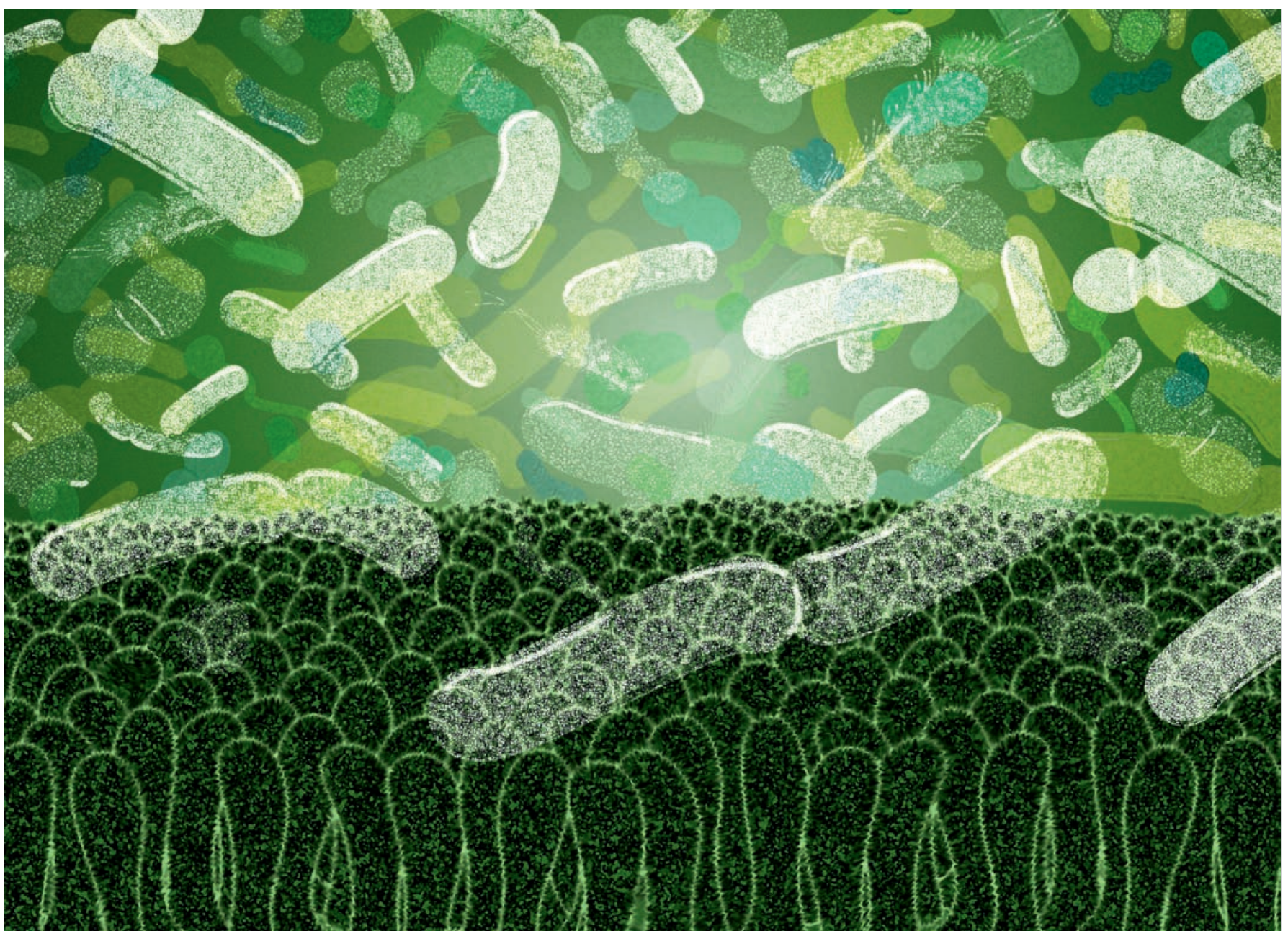

to govern many functions of metabolism and immunity.

Generally, the role of the microbiome can be summarized as mediating between the host and its environment. This implies a range of functions, from breaking down otherwise indigestible compounds such as cellulose, to modulating immune responses against both invasive pathogens and members of the microbial community itself. These functions, in turn, are controlled by signalling pathways that operate via receptors, primarily in the epidermal cells of the gut, through which the host interacts with the microbiota. Given the importance of these functions for the organism, the question arises as to whether the microbiome comprises specific or 'compulsory' genes that the organism must have for survival. If this is the case, it then becomes necessary to explain how the microbiome is acquired and maintained by the host in the first place.
Although research did not initially address these questions, scientists had nevertheless accumulated evidence that disruptions to the microbiome are either the cause of or are associated with a wide variety of conditions, particularly those involving inflammation and allergy, but also susceptibility to many infectious diseases. Clear links soon emerged with the two main forms of inflammatory bowel disease-ulcerative colitis and Crohn's disease-but also with several allergies, such as asthma and eczema, as well as metabolic conditions including obesity.

In some cases, these disruptions might involve or be caused by a bacterium that is not normally present or does not usually cause symptoms. The most celebrated example is Helicobacter pylori, which was identified in the early 1980s as the cause of most stomach and intestinal ulcers by Australians Robin Warren and Barry Marshall, who received the Nobel
Prize for Medicine in 2005 (Warren \& Marshall, 1984). Their discovery completely changed the understanding and treatment of ulcers, which were previously thought to be caused by stress or poor diet. The use of antibiotics in ulcer sufferers has since led to a dramatic reduction of both the condition itself and also the cancers that sometimes follow chronic or recurrent ulceration.

However, the vast majority of conditions associated with disruptions of the microbiome are tougher nuts to crack; they are rarely conveniently associated with specific microorganisms and the actual mechanisms involved have remained elusive. Nevertheless, the growing realization of the role of commensal microorganisms in health and disease has led to increased interest from both researchers and funders. The US National Institutes of Health, for instance, have begun a five-year, US\$115 million Human Microbiome Project to 
identify and analyse the human microbiota (http://nihroadmap.nih.gov/hmp).

A Ithough it is clear that certain changes in the microbiome are often associated with disease, it seems that the microbiota itself is in a constant state of flux, driven by variations in diet, environment and the immune status of the host. During the past year or so, however, scientists have collected evidence to indicate that although the microbiota is continuously changing, it does contain a constant subset of genes that might well be intrinsic to the host's well-being. A recent study at Washington University School of Medicine (St Louis, MO, USA) identified a core set of genes that seem to be present among the faecal microbiota of all humans, irrespective of the actual microorganisms involved. The study, conducted on 154 women, including identical and non-identical twins and their mothers, also indicated that deviations from this core microbiome-cases in which some of these core genes were lackingwere associated with various metabolic conditions, including obesity (Turnbaugh et al, 2009). This core microbiome, along with the wider peripheral microbiota, outnumbers human cells by ten to one. While bacteria seem to be the most common commensals, members of other domains can play significant roles, such as the methanomic Archaea that digest cellulose in ruminants to yield methane.

\section{Generally, the role of the microbiome can be summarized as mediating between the host and its environment}

Both the bacterial and archaeal members of human gut microbiota have been significantly affected by the use of antibiotics and high levels of hygiene, both of which can deplete the microbiome. As a result, certain conditions associated with microbiota disruption are much more prevalent in industrialized than in developing nations. Indeed, one result of modern medicine and hygiene is that some of the higher microorganisms in the human gut, including protozoa and parasitic worms, have virtually been eradicated in the industrialized nations, according to Rustam Aminov from the Gut Immunology Group at the University of Aberdeen in the
UK. This might sound like a good thing, but as Aminov pointed out, there is some evidence that the use of helminthic therapy_that is, giving a of dose helminth worms to patients - can alleviate the symptoms of Crohn's disease (Summers et al, 2005).

\section{...although the microbiota is continuously changing, it does contain a constant subset of genes that might well be intrinsic to the host's well-being}

More generally, there has been growing interest in the use of prebiotics, which contain micronutrients designed to restore normal microbiota populations; probiotics, which comprise the microorganisms themselves; and more specific antibiotics to fight undesirable organisms, either separately or in combination. As Aminov pointed out, there has been some success in treating ulcerative colitis with probiotics, at least to prevent remission, although he noted that "[t]rials of probiotics and prebiotics in Crohn's disease, however, are less convincing."

A s John Rawls at the University of North Carolina (Chapel Hill, NC, USA) commented, it will require more than an analysis of the genetic components of the microbiome to understand and efficiently manipulate it: "Our understanding of the Gl [gastrointestinal] ecosystem must be extended not only by development and implementation of molecular analytic methods, but also by in vitro culture systems that accurately mimic Gl tract environments as well as experimentally tractable mammalian and non-mammalian animal model systems that permit reductionist analyses of the Gl ecosystem."

Such models would enable scientists to assess the impact of different treatments in humans instead of using animal models-which is important given the huge inter-species differences in microbiota. New treatments are likely to evolve from the current broad-spectrum prebiotic and probiotic approaches into the more specific targeting of host receptors, according to Masashi Yanagisawa from the Howard Hughes Medical Institute at the University of Texas (Austin, TX, USA). "Currently the field is slowly moving to actually reveal the molecules that are designed to interface between microgenomes and the host," he said. "Certainly for drug delivery, new ways to modulate host receptors is interesting. Moderating gut microbes directly is very complex, and compared with that, moderating the receptor, either by agonising or antagonising, is much more specific."

Yanagisawa identified the short-chain fatty acid GPR41-a receptor in the colon that has a major role in nutrient absorption-as a promising target for drugs to treat obesity (Buck et al, 2008). "Blocking that receptor should reduce energy extraction efficiency on the host side," said Yanagisawa. "If that is the case, then antagonists to that receptor might be useful for reducing bodyweight."

Unfortunately, simply blocking a receptor is not always the solution. According to Aminov, the root causes of many if not most conditions that involve disruptions to the microbiota are genetic: "Environmental factors play a modulating role in the disease process," Aminov commented. "Nevertheless, these disorders are not thought to be caused by the environmental factors per se; they require genetic predisposition as a main factor in developing a disease."

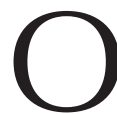
ne of the most studied examples is familial Mediterranean fever (FMF), which occurs largely among Jews, Armenians, Turks and Arabs, with a frequency as high as one in 250 people, according to Zaruhi Khachatryan from the Armenian Institute of Molecular Biology in Yerevan. FMF is associated with sporadic attacks of varying frequency, the symptoms of which include abdominal pain, pleurisy and leg joint pain. The causes of FMF are mutations in the Mediterranean fever gene (MEFV), which codes for the protein pyrin. Pyrin is known to interact with molecules involved in the inflammatory response, though its functions have not yet been fully characterized. Nevertheless, MEFV mutations cause symptoms of varying severity associated with characteristic changes in microbiota. "[W]e demonstrated that the specific structural changes in microbiota are primarily determined by the mutated MEFV gene," Khachatryan explained (Khachatryan et al, 2008). "This mutated gene may be associated with a dysregulated immune 
response to commensal bacteria [that do not harm the host], by generating defective signals on the host-gut microbiota signalling pathways."

\section{New treatments are likely to evolve from the current broad- spectrum prebiotic and probiotic approaches into the more specific targeting of host receptors...}

The various mutations in the MEFV gene amplify the host's immune response to commensal bacteria and reduce their number and diversity. As Khachatryan noted, the principal mechanism at work in these cases is innate immunity, which operates at mucosal surfaces through recognition of microorganism-associated molecular patterns (MAMPs). Recognition of a MAMP by the host occurs mainly through two major receptor systems called Toll-like (TLR) and Nod-like (NLR) receptors, which have a crucial role in regulating intestinal homeostasis, repair, angiogenesis and in controlling the number of commensal bacteria. "This signalling drives the synthesis of host antimicrobials, thus suppressing the overgrowth of bacteria at mucosal surfaces," Khachatryan said. "In Crohn's disease, for example, the failure [of the mutant NOD2 receptor] to recognize a bacterial ligand, muramyl dipeptide, [...] eventually results in the overgrowth of certain groups of bacteria at mucosal surfaces. This leads to the aggressive Th1mediated response against the commensal bacteria, resulting in diminished diversity." Th1 cells respond to such overgrowth by secreting cytokines that stimulate the production of cytotoxic T lymphocytes, which attack the bacteria.

In the case of FMF, the impact on the microbiome varies over time in step with the periodic nature of the disease. Bacterial diversity diminishes during acute phases but recovers during periods of remission. "The observed periodic changes are thought to be linked to inflammatory fluctuations in different stages of the disease," Khachatryan noted. "During an attack this may be explained by acute inflammation, whereas lowlevel sub-clinical inflammation conditions present in remission may account for the continuing immunoselection of gut microbiota."
$\mathrm{F}$ MF provides convincing evidence of the way in which an organism's genes and its immune status regulate the number and variety of its microbiota. Further evidence from animal models supports the idea that vertebrates aim to establish a core microbiome derived from fluctuating microbiota: a crucial subset of extragenomic microbial genes that can come from a variety of microorganisms. Rawls has studied reciprocal transplants of whole microbiota populations between zebrafish and mice, using gnotobioticgerm-free-individuals as recipients to study host responses to supposedly alien microbial populations (Rawls et al, 2006). Although mammalian and fish digestive systems are populated by unrelated bacteria from different phyla, the microbiota from one seem to work reasonably well in the other after a microbiotic transplant. "Whereas the mammalian gut microbiota is dominated by members of the Firmicutes and Bacteroidetes, the intestines of zebrafish are dominated by Proteobacteria and Fusobacteria," Rawls said. "Interestingly, these compositionally-distinct microbial communities elicit similar responses from their respective hosts. Moreover, they can elicit the same responses when transplanted into the reciprocal host. These results suggest that there is a striking degree of functional redundancy among compositionally-distinct microbial communities."

\section{There is, for example, an almost complete lack of knowledge about the role of viruses, which are also present in huge abundance in the gut}

This functional redundancy is a feature of microbiomes in general, but the impact of transient components of the microbiota should not be overlooked either, as they have a large role in the ever changing biochemistry of the gut, and so potentially in disease, according to Jeremy Nicholson, head of biological chemistry at Imperial College, London, UK. "I think that there are both stable microbiome components and unstable ones, with the former changing only over long periods associated with ageing, and therefore with changing host biology," he said. "The latter changes more with diet and stressors."

\section{...it is likely that the virome will turn out to be an important part of the overall microbiome, with significant scope for therapeutic development}

This varying state is determined by a combination of factors. Host genetics might determine the functional composition, but the overall biochemical affect can also be shaped by other things, including the gut environment during microbiome acquisition and just after birth. "The bug set you get at birth may determine the development of later ecology," Nicholson explained, noting that while the microbiome is not strictly speaking inherited, the mother has a significant effect on its composition, as does the mode of birth. "Animals from any species that are born by caesarean section are generally not very well and grow poorly," he said.

M uch remains to be discovered about the factors that govern the acquisition of the microbiome, and its subsequent development and maintenance by the host. There is, for example, an almost complete lack of knowledge about the role of viruses, which are also present in huge abundance in the gut. "Horizontal gene transfer among intestinal bacteria is thought to be rampant, and it is likely that many of these transfer events are mediated by viruses," Rawls commented. "However, empirical data supporting these notions is sparse." It is also uncertain whether viruses play a significant, direct role in diseases mediated by the microbiome, rather than a long-term role in evolution.

Phages could have an important role in future therapies that target the microbiome (Dominguez-Bello \& Blaser, 2008). Phages could be probiotics in the sense that they are microorganisms themselves, or they could act as prebiotics by infecting certain bacteria and therefore encouraging proliferation of others. In addition, they might also play an antibiotic role by attacking undesirable bacteria. While the era of phage biotics is at a very early stage, it is likely that the virome will turn out to be an important part of the overall microbiome, with significant scope for therapeutic development. Notwithstanding the target or the means, it will certainly require many more years of research to 
understand how the microbiome interacts with its host and to develop new therapeutics that are much more specific than crude broad-spectrum antibiotics.

\section{REFERENCES}

Buck S et al (2008) Effects of the gut microbiota on host adiposity are modulated by the shortchain fatty-acid binding $G$ protein-coupled receptor, Gpr41. Proc Natl Acad Sci USA 105: 16767-16772
Dominguez-Bello MG, Blaser MJ (2008) Do you have a probiotic in your future? Microbes Infect 10: 1072-1076

Khachatryan ZA, Ktsoyan ZA, Manukyan GP, Kelly D, Ghazaryan KA, Aminov RI (2008) Predominant role of host genetics in controlling the composition of gut microbiota. PLOS ONE 3: e3064

Rawls JF, Mahowald MA, Ley RE, Gordon JI (2006) Reciprocal gut microbiota transplants from zebrafish and mice to germ-free recipients reveal host habitat selection. Cell 127: 423-433
Summers RW, Elliott DE, Urban JF, Thompson R, Weinstock JV (2005) Trichuris suis therapy in Crohn's disease. Gut 54: 87-90

Turnbaugh PJ et al (2009) A core gut microbiome in obese and lean twins. Nature 457: 480-484

Warren JR, Marshall BJ (1984) Unidentified curved bacilli in the stomach of patients with gastritis and peptic ulceration. Lancet 1: 1311-1315

\section{Philip Hunter}

doi:10.1038/embor.2009.204

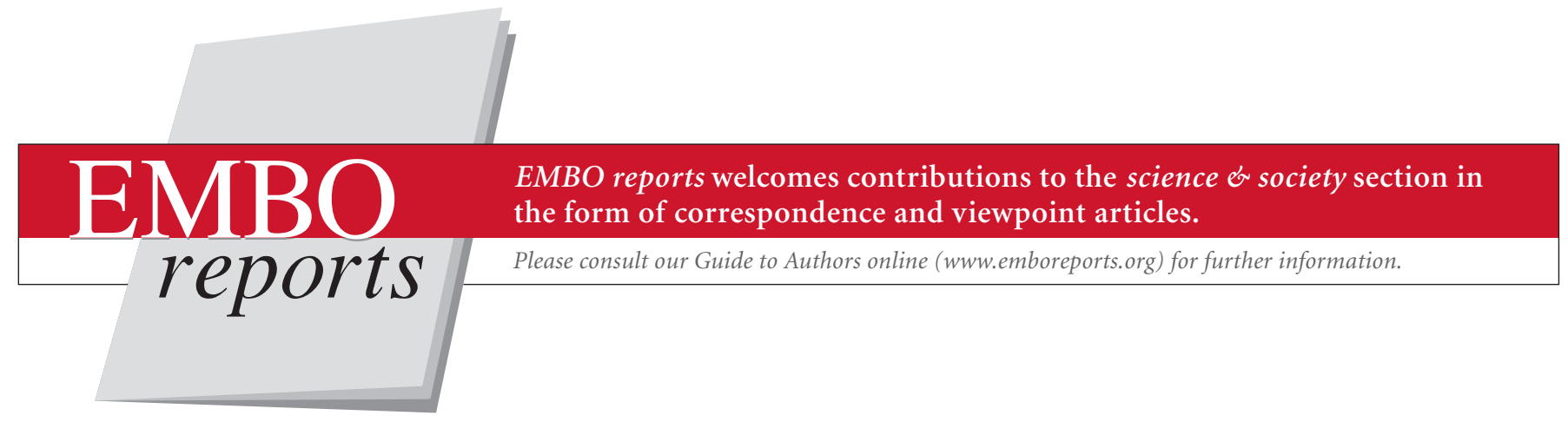

